\title{
Téoros
}

Revue de recherche en tourisme

\section{Nature et tourisme}

L'écotourisme au Québec en 2002

\section{Maurice Couture}

Volume 21, numéro 3, automne 2002

Écotourisme

URI : https://id.erudit.org/iderudit/1072502ar

DOI : https://doi.org/10.7202/1072502ar

Aller au sommaire du numéro

Éditeur(s)

Université du Québec à Montréal

ISSN

0712-8657 (imprimé)

1923-2705 (numérique)

Découvrir la revue

Citer cet article

Couture, M. (2002). Nature et tourisme : l'écotourisme au Québec en 2002.

Téoros, 21(3), 43-49. https://doi.org/10.7202/1072502ar

Ce document est protégé par la loi sur le droit d'auteur. L'utilisation des services d'Érudit (y compris la reproduction) est assujettie à sa politique d'utilisation que vous pouvez consulter en ligne.

https://apropos.erudit.org/fr/usagers/politique-dutilisation/
Cet article est diffusé et préservé par Érudit.

Érudit est un consortium interuniversitaire sans but lucratif composé de l'Université de Montréal, l'Université Laval et l'Université du Québec à Montréal. Il a pour mission la promotion et la valorisation de la recherche. https://www.erudit.org/fr/ 


\section{Nature et tourisme}

L'écotourisme au Québec en 2002

\section{Maurice Coułure}

$\mathbf{L}$ e document intitulé Nature et tourisme : L'écotourisme au Québec en 2002 est le fruit d'une démarche initiée par Tourisme Québec au cours de 2002, qui s'insère dans un processus devant mener à la production, au cours de 2003, d'un plan de développement et de promotion de cette forme de tourisme au Québec. Une table de concertation sur l'écotourisme formée de représentants privés, régionaux, gouvernementaux et du milieu universitaire a apporté une contribution importante à cet exercice de réflexion. Le présent article présente sommairement le diagnostic stratégique de l'écotourisme au Québec qui a été réalisé par l'auteur.

\section{Popularité croissante de l'écotourisme}

La démarche québécoise s'est, du point de vue théorique, fortement inspirée des caractéristiques retenues par l'Organisation mondiale du tourisme (OMT) pour cette forme de tourisme. L'écotourisme est ici défini comme :

- un tourisme de nature principalement axé sur l'observation et l'appréciation de la nature et des composantes culturelles qui y sont associées;

- offert et pratiqué de façon durable (retombées économiques pour les résidants et respect du milieu d'accueil et de l'environnement naturel / culturel) ;

- auquel se superposent les éléments distinctifs de l'écotourisme, tels que contribution particulière aux efforts de protection, participation du milieu d'accueil et composantes éducatives (ex. : interprétation).

Cette forme de tourisme gagne en popularité au Québec avec l'émergence de projets dans la plupart des régions touristiques, la volonté de nombreuses entreprises de créer dans un avenir rapproché des nouveaux produits écotouristiques et les diverses initiatives de partenariat visant le développement d'outils pour ce secteur.

\section{Enjeux et défis :}

- Partage de paramètres communs en matière d'écotourisme parmi les intervenants privés, associatifs et gouvernementaux intéressés, afin d'éviter le galvaudage potentiel du terme « écotourisme »;

- Reconnaissance et intégration de l'écotourisme dans les outils de planification gouvernementale et les stratégies de développement local et régional.

\section{Immensité, diversité et nordicité}

L'immense territoire québécois - environ 1,7 million de kilomètres carrés - se caractérise par : sa nordicité et ses importants bassins hydrographiques; des ensembles géographiques aux images fortes ; une diversité des écosystèmes et des régions géologiques, de la faune, de la flore et de nombreux phénomènes naturels saisonniers. Cette diversité s'observe aussi par les composantes culturelles d'hier et d'aujourd'hui présentes à proximité ou à l'intérieur de nombreux sites naturels : patrimoine culturel et humain et bâti varié ; personnalité francophone dominante qui prend des formes diverses selon les régions ; diversité culturelle présente sur l'ensemble du territoire (pensons, notamment, à la présence des nations autochtones).

En contrepartie, le développement de l'écotourisme doit se réaliser dans divers contextes : plusieurs paysages naturels ont perdu de leur attrait faute de protection ; de nombreux sites naturels sont particulièrement fragiles; plusieurs plans et cours d'eau sont pollués et leurs berges privatisées ; la saison hivernale offre une diversité moindre sur le plan des possibilités d'observation de la faune et de la flore; la diversité faunique et floristique du Québec est moins importante que celle de certaines destinations qui disposent de climats tropicaux ou subtropicaux.

Le Québec dispose sur son territoire d'atouts importants pour supporter le développement d'expériences d'écotourisme très variées, développement qui peut mettre à l'avant-scène l'immensité, la diversité et la nordicité du Québec, ainsi que ses bons potentiels d'observation de la nature, en plus de miser sur des produits jumelant découverte de la nature, de la culture et des diversités régionales dans une approche « quatre saisons ».

\section{Milieux naturels : matière première de l'écotourisme}

Les territoires désignés comme aires naturelles protégées - reconnues comme telles selon les catégories de l'Union mondiale pour la nature - se révèlent plus particulièrement propices à l'offre de produits d'écotourisme concurrentiels de qua- 
lité : territoires naturels peu perturbés, présence d'attributs écologiques ou esthétiques particuliers, réglementation des activités limitant les conflits d'usage, protection par rapport aux activités d'exploitation ou d'utilisation du territoire (ex. : foresterie, mines). Bref, il s'agit d'actifs durables pour lesquels il est possible d'investir dans la mise en valeur et la protection, en développant des produits écotouristiques et en les positionnant sur les marchés.

\section{Enjeux et défis :}

- Adoption de mesures de protection des paysages qui présentent un intérêt particulier;

- Protection et mise en valeur des composantes culturelles des environnements naturels et identification des aspects culturels et historiques les plus intéressants à des fins de mise en valeur et d'interprétation;

- Exploitation des possibilités liées à la grande diversité des composantes naturelles, culturelles et des phénomènes saisonniers du Québec dans la création de produits.

Les aires protégées demeurent peu développées dans la partie méridionale, la forêt boréale et les milieux marins du Québec et peu de mécanismes permettent de protéger les rivières et les corridors naturels qui les entourent. Mais le Québec a fait un important gain en juillet 2002 avec l'ajout de onze territoires mis en réserve sur la CôteNord et de six projets de parcs nationaux dans le Nord du Québec. L'ajout de ces territoires fait passer de $2,91 \%$ à $4,85 \%$ la proportion protégée du territoire québécois. L'objectif du Gouvernement québécois est de passer à $8 \%$ d'ici 2005, pour rejoindre la moyenne nationale de nombreux autres territoires.

\section{Enjeux et défis :}

- Protection d'échantillons représentatifs de l'ensemble de la biodiversité du Québec et diversification des types d'aires protégées;

- Accroissement des aires protégées - en nombre et en superficie - du côté de la forêt boréale, du Québec méridional, des milieux marins et des rivières;

- Partenariat entre intervenants de l'écotourisme et organisations environnementales;

- Engagement du secteur de l'écotourisme dans les efforts de préservation;

- Recours à l'écotourisme en tant qu'outil d'éducation auprès des québécois afin de supporter la vision de la stratégie québécoise sur la biodiversité (« ...les Québécois doivent s'imprégner davantage d'une culture écologique et évoluer en plus grande harmonie avec la natu$\left.r e^{1} \gg\right)$.

Les parcs nationaux figurent parmi les aires protégées les plus prisées des clientèles. Les vingt-quatre parcs nationaux du Québec (des réseaux fédéral et québécois), auxquels s'ajoute le Parc marin Saguenay-Saint-Laurent (PMSSL), représentent une superficie totale de 7400 kilomètres carrés. Or, la fréquentation totale du réseau des parcs nationaux se concentre spécifiquement à quelques parcs. D'autres types d'aires protégées - de petite taille pour la plupart - servent actuellement ou pourraient servir de base à des produits d'écotourisme : réserves écologiques, réserves nationales de faune, refuges d'oiseaux migrateurs, refuges fauniques, sites protégés par d'autres organismes privés, « écosystèmes forestiers exceptionnels » (EFE). Plusieurs ont un caractère exceptionnel et offrent de bonnes possibilités d'observation de la nature et d'excellentes possibilités d'interprétation, alors que quelques-unes bénéficient aussi d'une reconnaissance internationale, ce qui peut se révéler avantageux sur le plan du marketing.

Des facteurs comme la facilité d'accès, la proximité des marchés, la concentration de la demande à certaines périodes (été, fins de semaine) et la popularité de certaines activités peuvent créer des pressions importantes sur les aires protégées. À titre d'exemple, le PMSSL - pôle majeur pour les activités d'observation des baleines - a dû mettre en place, en raison de la pression qui résulte de la popularité de l'activité, une réglementation encadrant les activités d'ob- servation en mer qui y sont offertes et limitant le nombre de permis destinés aux croisières d'observation.

\section{Enjeux et défis :}

- Nécessité de fixer des seuils à ne pas dépasser en ce qui concerne l'achalandage et les limitations des activités permises, afin d'assurer la protection des sites et, par extension, la qualité des expériences d'écotourisme qui peuvent s'y dérouler;

- Développement des connaissances, aménagement intégré et préservation de la valeur et de l'intégrité des territoires à fort potentiel de développement;

- Adhésion aux pratiques de conservation et d'éducation de l'écotourisme, de la part des entreprises qui désirent opérer dans une aire protégée;

- Participation du secteur de l'écotourisme aux efforts de protection du patrimoine naturel : développement des connaissances, adoption de mesures de suivi et d'évaluation (mesures des impacts, monitoring, indicateurs).

Le Québec compte de nombreux territoires non désignés comme aires naturelles protégées. Plusieurs de ceux-ci offrent quand même ou peuvent offrir de bonnes possibilités pour des activités d'écotourisme : possibilités d'observation de la faune, mise en application de principes de développement durable, vaste réseau d'hébergement en forêt, proximité de parcs nationaux. En contrepartie, ces territoires sont soumis aux aléas d'autres types d'exploitation et sont très susceptibles d'être exposés aux conflits d'usage (chasse, plein air « motorisé », foresterie, mines, villégiature « privée ») et ne peuvent garantir la constance ni la pérennité de leurs attributs naturels. Il s'agit de territoires situés dans le Québec méridional, notamment : les réserves fauniques, les territoires des pourvoiries à droits exclusifs et ceux des ZEC (zones d'exploitation contrôlée), les forêts modèles et les forêts habitées, les centres éducatifs forestiers, les parcs régionaux d'importance et les terrains sous baux ou vendus par le ministère des Ressources naturelles (MRN) pour des projets récréotouristiques. 
De leur côté, les trois réserves mondiales de la biosphère du Québec (Charlevoix, lac Saint-Pierre et mont Saint-Hilaire) sont des territoires reconnus, à l'échelle internationale, qui présentent de bonnes possibilités pour l'écotourisme, autant en raison de leur environnement que du fait qu'ils constituent des laboratoires de développement durable.

Un profil partiel démontre que certains sites naturels de qualité et «points chauds »-comme le fleuve Saint-Laurent et le PMSSL, les parcs nationaux ainsi que certaines réserves fauniques et rivières sont utilisés par les producteurs de tourisme de nature et d'écotourisme.

Force est de reconnaître que plusieurs sites ne sont pas exclusifs à l'écotourisme et que les milieux naturels doivent souvent être partagés entre des expériences desservant des clientèles aux aspirations souvent très différentes. Conséquemment, des conflits d'usage surgissent lors de la présence sur un même territoire d'activités touristiques incompatibles : appréciation et observation de la nature versus prélèvement faunique (chasse et la pêche) ou plein air " motorisé » axé sur la performance et la vitesse.

\section{Enjeux et défis :}

- Accroissement des partenariats publics / privés dans les domaines de l'aménagement et de l'accessibilité aux territoires, de la protection des milieux naturels et du développement de produits;

- Identification et implantation de mécanismes d'harmonisation des usages sur les terres publiques;

- Reconnaissance de la dualité et de la complémentarité entre «tourisme de nature » durable et «écotourisme »: le caractère durable des environnements naturels utilisés et la qualité des expériences d'écotourisme offertes dépendent des bonnes pratiques et d'une vision tenant compte de toutes les activités de tourisme qui se déroulent en milieu naturel.

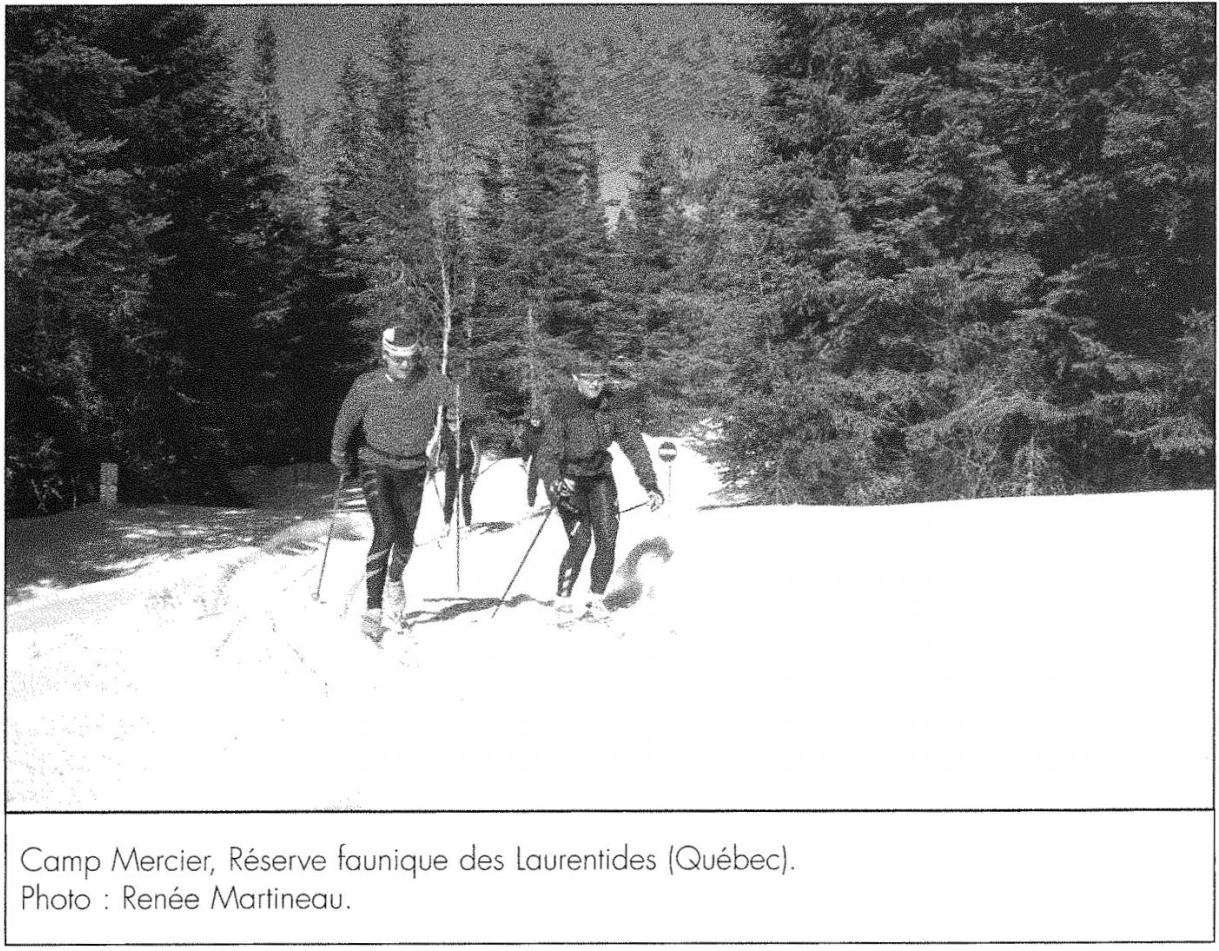

\section{Sentiers et corridors d'accès à la nature}

Les corridors d'accès à la nature ou reliant différents sites naturels, tels que sentiers pédestres, voies cyclables, parcours canotables ou sentiers de ski de fond et de raquette en milieu naturel, jouent ou sont appelés à jouer un rôle important dans l'offre de produits d'écotourisme. Le Québec peut aussi compter sur le développement de grands réseaux de sentiers - comme le Sentier national, le Sentier international des Appalaches, le Sentier transcanadien et la Route verte - qui peuvent s'intégrer à des circuits de découverte ou à des produits amalgamant aventure, cyclotourisme et écotourisme. La pérennité des sentiers n'est toutefois pas garantie et ils ne sont pas à l'abri des conflits d'usage.

\section{Enjeux et défis :}

- Développement et contrôle des réseaux d'accès appropriés aux expériences d'écotourisme (sentiers, cours d'eau navigables, vélo, etc.);

- Protection des territoires récréotouristiques déjà utilisés sur les terres publiques et création de liens entre produits d'écotourisme et grands réseaux de sentiers.

\section{Produits et organisations}

Les produits de tourisme de nature et d'écotourisme offerts au Québec dépendent d'une multitude d'intervenants qui jouent des rôles variés. Aux 1040 intervenants / établissements qui prennent directement part à l'offre de produits de tourisme de nature et d'écotourisme s'ajoutent de nombreux autres impliqués dans des services complémentaires : hébergement, transport, restauration, forfaitisation. Aucune étude n'existe sur l'importance économique réelle générée par les intervenants du tourisme de nature et de l'écotourisme.

L'offre permet de soutenir des activités variées s'adressant autant à des clientèles qui désirent effectuer des visites individuelles autoguidées qu'à celles qui veulent participer à des sorties guidées en petits groupes, avec ou sans hébergement. Malgré l'existence de produits bien structurés, la qualité est inégale et les produits intégrés demeurent sous-développés.

Outre l'offre liée aux gestionnaires de territoires, on trouve :

- des producteurs de tourisme de nature, d'aventure et d'écotourisme correspondant principalement à de jeunes et peti- 
tes entreprises saisonnières qui offrent des excursions de courte durée et qui se segmentent entre : les «producteurs spécialisés » $(10 \%)$, qui se consacrent exclusivement à l'offre de produits d'écotourisme, et les « producteurs mixtes » (67\%), qui offrent au moins une activité d'écotourisme parmi d'autres activités d'aventure ou de plein air;

- quelques pourvoiries qui offrent des produits à caractère écotouristique basés sur les diverses possibilités d'observation des ressources naturelles (130 pourvoiries offrent l'observation de la faune selon la FPQ - Fédération des pourvoyeurs de Québec);

- quelques tour opérateurs / voyagistes spécialisés localisés à l'extérieur du Québec et offrant des produits axés sur les croisières d'observation et de découverte du Saint-Laurent, l'observation de la nature, des oiseaux, des baleines et des blanchons et les visites de parcs nationaux.

L'offre est dominée par des «éco-aventures », soit un jumelage d'activités d'aventure douce et d'observation / interprétation de la nature / culture d'un milieu donné. Les expériences hivernales d'écotourisme apparaissent comme peu répandues malgré de bonnes possibilités. Plusieurs produits d'écotourisme dépendent de la facilité d'observer avec succès certaines espèces animales ou certains phénomènes naturels, c'est-à-dire de facteurs naturels impondérables et incontrôlables. Il s'agit de produits difficiles à garantir et à promouvoir, d'année en année, selon un calendrier fixe.

Sur le plan culturel, les produits d'écotourisme offerts dans les parcs nationaux s'appuient sur diverses thématiques historiques et patrimoniales. Cet aspect est omniprésent dans le cas des entreprises autochtones (traditions, artisanat) et surpasse même l'importance accordée aux milieux naturels utilisés. Le caractère culturel des milieux naturels apparaît comme sous-évalué et demeure moyennement répandu auprès des producteurs, où l'on trouve notamment diverses imitations de produits autochtones. Notons finalement que plusieurs des plus beaux villages du Québec sont situés à proximité de milieux naturels et peuvent jouer le rôle à la fois d'attrait culturel, d'étape et de lieu de services dans un circuit d'écotourisme.

\section{Enjeux et défis :}

- Diversification des produits, raffinement de la composante culturelle liée aux milieux naturels visités et développement de l'écotourisme hivernal et automnal;

- Mise en valeur de la diversité culturelle québécoise, notamment l'identité culturelle autochtone dans une approche misant sur l'authenticité;

- Adoption d'approches de « marketing responsable » en créant des attentes réalistes auprès des clients (ex. : réelles possibilités d'observation de la faune) et en fournissant des renseignements justes et véridiques sur les expériences offertes.

\section{Mise en pratique de l'écotourisme}

Les activités d'éducation et de sensibilisation auprès des clientèles s'intègrent à différents moments du séjour d'un touriste et visent à encourager des comportements responsables avant le départ ou dès l'arrivée à destination. Selon l'analyse des sites Internet des producteurs spécialisés, il s'agirait d'une forme de communication sous- exploitée. À l'opposé, les producteurs à but non lucratif qui ont une mission de conservation ou scientifique ont, dans la plupart des cas, instauré des codes de conduite pour leurs clientèles (ex. : Société Duvetnor). À ce chapitre, parmi une série d'initiatives, soulignons (Couture, 2002): le Code de bonnes pratiques de l'écotouriste ${ }^{2}$ proposé par Tourisme Québec ; le site Internet «Baleines en direct ${ }^{3}$ »; le guide $L a$ découverte de la faune et des habitats en pourvoirie; ainsi que le Projet de norme de qualité du Bureau de la normalisation du Québec (BNQ) sur les produits d'écotourisme prévoyant des actions précises en matière de sensibilisation et d'éducation des clientèles, ce qui devrait contribuer à répandre un tel type de pratiques parmi les entreprises.

Les activités de sensibilisation du milieu d'accueil semblent, à partir des renseignements disponibles, encore très peu développées chez les producteurs. Cette responsabilité serait assumée par certains gestionnaires de territoires qui offrent des activités de sensibilisation à la protection de l'environnement ou permettent l'engagement de la population locale (comité de coordination du PMSSL, tables d'harmonisation dans chacun des pares sous la responsabilité de la Sépaq - Société des établissements de plein air du Québec).

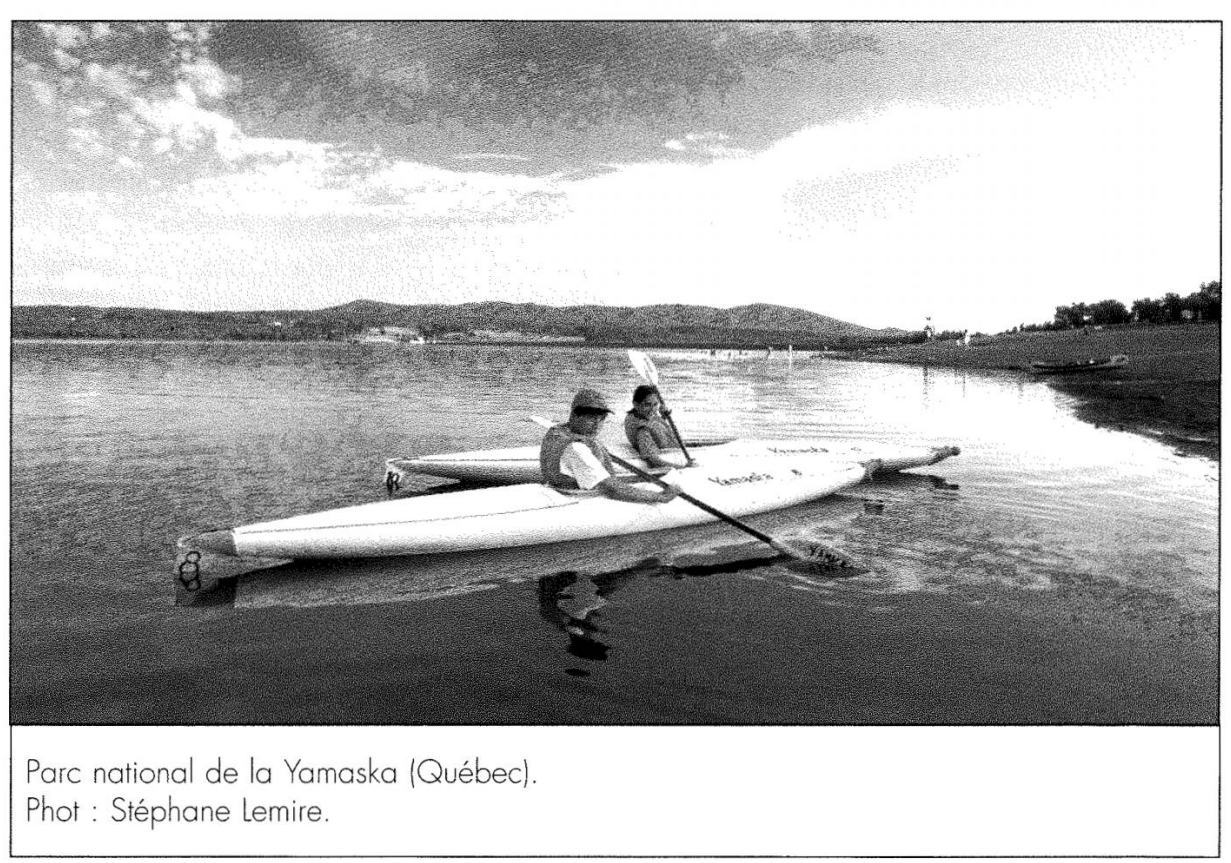


Dans le contexte de l'écotourisme, les activités d'éducation portant sur les milieux visités prennent, dans la majorité des cas, la forme d'activités d'interprétation. Celles-ci favorisent 1'apprentissage, aident les clients à développer une meilleure compréhension des milieux visités, font la promotion de comportements appropriés et servent aussi à enrichir l'expérience offerte aux clients. Parmi les caractéristiques des activités d'éducation et d'interprétation soulignons qu'elles sont :

- présentes dans les parcs nationaux, compte tenu de leur mission éducative ; leurs gestionnaires ont développé une expertise reconnue;

- répandues dans la plupart des réserves fauniques;

- relativement répandues parmi les producteurs sous la forme d'activités d'observation du milieu naturel, d'aventures et de plein air jumelées à des activités d'interprétation;

- offertes par certaines pourvoiries sous la forme de produits plus spécialisés d'observation de la faune.

Mais la nature, la portée et la qualité des activités d'interprétation offertes dans les pourvoiries ou par les producteurs du secteur demeurent peu documentées. Selon The International Ecotourism Society ${ }^{4}$ (TIES), l'interprétation constitue, en ce qui concerne la qualité, le maillon faible des activités d'écotourisme offertes par les entreprises d'écotourisme dans le monde. Le Projet de norme de qualité sur les produits d'écotourisme apporte une grande attention à la rigueur des plans d'interprétation et devrait concourir à améliorer l'offre dans le domaine.

Dans le contexte québécois, les avantages économiques pour les communautés d'accueil de tourisme de nature et d'écotourisme s'appuient sur le fait qu'une majorité de producteurs sont en fait des entrepreneurs locaux qui ont leurs racines dans les diverses régions du Québec, y concentrent leurs activités et contribuent à l'économie locale. Les retombées économiques liées aux dépenses des touristes, aux investissements, aux emplois et aux achats locaux générés par des parcs na- tionaux, des réserves fauniques et d'autres milieux naturels sont aussi très importantes pour les régions.

Les pratiques environnementales des entreprises demeurent relativement limitées et consistent notamment à récupérer ou à ramener ses déchets et à ne pas piétiner ou faire de prélèvement de plantes ou de fruits lors des activités en milieu naturel. Plusieurs initiatives visent à outiller les entreprises, par exemple le «Programme qualité » de Tourisme Québec, en prévoyant l'adoption de pratiques environnementales. Les pratiques durables ne sont que peu mises en œuvre dans les services d'hébergement, de restauration et de transport ; elles rendent difficile la création de forfaits dont toutes les composantes seraient conformes aux valeurs de l'écotourisme.

Les contributions de la part des entreprises à la préservation de l'environnement prennent des formes diverses. Elles sont bien entendu bien tangibles dans le cas des organismes qui ont une vocation première de conservation ou de recherche à laquelle s'est juxtaposée une vocation d'écotourisme et prennent les formes suivantes : engagement dans des causes environnementales ; gestion durable du territoire et de la faune ; collaboration et participation à diverses démarches de cueillette d'information, de sensibilisation ou de surveillance (ex. : Réseau ObservAction de la Biosphère, Réseau d'observation des mammifères marins du Bas-Saint-Laurent, Attention Fragîles).

\section{Enjeux et défis :}

- Adoption généralisée, de la part des intervenants du secteur, d'approches de développement et de gestion maximisant les retombées locales;

- Approches privilégiant l'engagement et la responsabilisation des milieux d'accueil dans le développement de l'écotourisme;

- Évaluation des programmes d'interprétation actuels offerts par les entreprises;

- Implantation de la norme de certification des produits en tant qu'outil de gestion de la qualité et de support à une offre écotouristique authentique de calibre international;

- Défi de créer des forfaits qui jumellent des prestations d'écotourisme de qualité à un ensemble d'entreprises de services qui ont des pratiques de tourisme durable.

\section{Demande de produits d'écotourisme}

Les principales caractéristiques des marchés peuvent se résumer comme suit :

- Les écotouristes expérimentés effectuant des voyages où l'écotourisme est le principal but de voyage représentent une niche de marché (moins de $5 \%$ du marché mondial), mais seraient en croissance ;

- La plus grande partie de la demande se compose :

1. d'écotouristes occasionnels : un segment qui consomme des activités comme l'appréciation de la nature, la visite de parcs nationaux, l'observation de la faune et de la flore dans le cadre d'un voyage qui, dans la majeure partie des cas, n'a pas pour principal but l'écotourisme ou le tourisme de nature ;

2. d'écotouristes potentiels / touristes de nature « à convertir aux valeurs de l'écotourisme » : soit les clients susceptibles de s'intéresser à l'écotourisme, compte tenu de leur intérêt pour des voyages axés sur la nature.

Ces écotouristes proviennent de marchés sur lesquels le Québec est déjà actif (les provinces canadiennes, les États-Unis, l'Europe).

Le réseau de distribution est caractérisé par le rôle dominant des spécialistes. C'est ce dernier type de tour opérateurs (TO) qui est le plus actif dans l'organisation et la vente de voyages de tourisme de nature et d'écotourisme. Certains TO travaillent de concert avec des producteurs québécois ou avec des réceptifs spécialisés du Québec qui sont apparus au cours des dernières années. Des organismes renommés en conservation (dont la Société Audubon) ont pris 
le virage de l'écotourisme et offrent à leurs membres des voyages éducatifs respectueux de l'environnement. Finalement, le réseau régulier des grossistes et des agences demeure impliqué compte tenu de l'intégration de courtes activités de découverte de la nature à des circuits touristiques traditionnels.

On connaît mal le marché des écotouristes québécois, mais certaines données - notamment leur participation à des activités d'observation de la faune - laissent présager l'existence d'un marché intérieur possible non négligeable pour des activités d'appréciation de la nature et, par extension, des produits d'écotourisme. Certaines données d'achalandage sur l'observation des baleines, les visites dans les parcs nationaux effectuées par des touristes et les clientèles des producteurs de tourisme de nature, d'aventure et d'écotourisme démontrent l'importance des marchés internationaux pour ces produits au Québec.

À la demande croissante d'expériences de nature / aventure / écotourisme s'ajoute la croissance prévisible de la demande de certains produits pouvant être exploités avec succès au Québec :

- croisières de découverte de la nature avec des embarcations de petite et moyenne capacité ; secteur de l'observation des baleines;

- expériences jumelant nature et culture autochtone ; existence de plusieurs marchés de proximité demeurant encore largement sous-exploités par les producteurs (Ontario, États américains limitrophes);

- possibilité de capitaliser sur la réputation internationale bien établie du Canada en tant que destination nature et de destination « sécuritaire »;

- contexte de sur-affluence des parcs nationaux américains pouvant se traduire par un intérêt de la part des Américains pour les parcs nationaux du Québec.

\section{Enjeux et défis :}

- Efforts devant à la fois viser, avec une offre adaptée, les « écotouristes convaincus », à la recherche d'expériences au- thentiques, de qualité, certifiées et spécialisées et les " écotouristes potentiels » à convaincre (touristes de nature) et à sensibiliser à certaines valeurs de l'écotourisme;

- Définition d'approches de mise en marché et de promotion adaptées aux réalités d'intervenants variés, à de nombreux types de clientèles et à la nécessité de promouvoir des comportements responsables auprès des clientèles;

- Défi de positionner l'écotourisme à l'échelle d'une destination - ensemble du Québec ou d'une région touristique dans un contexte où il faut à la fois positionner d'autres produits qui pourraient être incompatibles;

- Amélioration des connaissances sur les TO internationaux spécialisés qui vendent ou sont susceptibles de vendre les produits du Québec et identification des approches visant à les sensibiliser aux valeurs et aux possibilités de développement de l'écotourisme au Québec;

- Développement et intégration de portions d'écotourisme à des produits davantage traditionnels pour satisfaire un marché majoritairement composé de touristes jumelant l'écotourisme à d'autres motifs de voyage;

- Adaptation de l'offre à une clientèle qui vieillit, qui recherche des expériences de qualité, qui peut avoir des besoins très spécialisés et qui voyage de plus en plus sur une base individuelle.

\section{Contexte concurrentiel}

Les produits québécois de tourisme de nature et d'écotourisme sont soumis à une forte concurrence internationale et à l'offre de pays bien positionnés par leur exotisme. Outre la concurrence provenant des pays en développement, plusieurs destinations européennes constituent aussi des concurrents de taille pour le Québec, d'autant qu'ils peuvent compter sur des composantes culturelles et patrimoniales distinctes et bien développées liées à des milieux naturels spectaculaires (Alpes, Fjords).

En ce qui concerne l'observation des baleines - produit où le Québec est bien présent -, le nombre de destinations offrant ce type d'expérience n'a cessé de croître. Quelques États de la Nouvelle-Angleterre constituent ici des concurrents sérieux.

Le Québec n'est pas du tout positionné parmi les destinations proposées aux membres d'organismes de conservation ou par des sites Internet spécialisés en écotourisme. Ici, la concurrence vient principalement de pôles bien établis, de produits qui misent sur des parcs nationaux et des environnements nordiques très variés (Alaska, Sibérie, Laponie, Islande, Sibérie, Kamtchatka).

Pour le grand marché du tourisme de nature, les États-Unis peuvent notamment compter sur leur réseau reconnu de parcs nationaux. Au Canada, la concurrence vient de la Colombie-Britannique, du Manitoba, de l'̂̂le-du-Prince-Édouard et de la Saskatchewan qui s'intéressent particulièrement à l'écotourisme, mais aussi des provinces de l'Atlantique et de l'Ontario qui ont fait des gains spectaculaires en aires protégées au cours des dernières années.

\section{Enjeux et défis :}

- Développer des produits d'écotourisme certifiés, de calibre international, concurrentiels, jumelant milieux naturels exceptionnels, activités bien structurées d'apprentissage et d'interprétation et séjours intégrant plusieurs activités;

- Évaluation des potentiels de forfaitisation pour le secteur de l'écotourisme basée sur le recours au réseau existant d'hébergement en forêt ou aux services de tourisme rural et d'agrotourisme, l'intégration de centres d'interprétation axés sur la nature / culture d'une région, le jumelage de prestations d'écotourisme et d'activités de randonnées diverses et la création d'itinéraires thématiques et de circuits interprovinciaux;

- Évaluation des moyens permettant aux produits d'écotourisme québécois d'être identifiés sur les sites Internet de référence majeurs ou les sites Internet commerciaux d'écotourisme, ainsi que sur les sites des organismes de conservation. 


\section{Organisation du secteur}

Le développement du secteur dépend et bénéficie de l'engagement d'une grande variété d'intervenants et peut compter sur une série d'organismes dynamiques bien structurés. On fait ici référence aux regroupements d'intervenants (ex. : Aventure Écotourisme Québec, Association des croisières-excursions du Québec, FPQ Fédération des pourvoyeurs de Québec, STAQ - Société touristique des Autochtones du Québec, fédérations de plein air) ; aux organisations de conservation intéressées à des dossiers comme les aires protégées ; aux organismes qui ont une expertise dans la préservation et la mise en valeur du Saint-Laurent ; aux conseils régionaux de l'environnement (CRÉ) et aux organismes des domaines de l'expertise et de la formation (ex. : Association québécoise d'interprétation du patrimoine, Association québécoise pour la promotion de l'éducation relative à l'environnement, institutions collégiales et universitaires offrant des cours spécifiques, entre autres, en écotourisme, en tourisme, en environnement, en écologie; divers groupes de recherche universitaires engagés dans les secteurs du tourisme, de l'environnement et du développement durable).

Par ailleurs, en ce qui concerne le développement des ressources humaines, le Québec peut compter globalement sur une main-d'œuvre compétente, mais son recrutement en région demeure problématique. Plusieurs institutions de formation dans tout le Québec sont intéressées au tourisme de nature et à l'écotourisme et peuvent contribuer au développement des compétences particulières requises par cette forme de tourisme (écologie, interprétation, gestion durable, etc.).

Les efforts de planification actuels ont trait à divers outils de référence ainsi qu'à des stratégies existantes qui ont une incidence sur l'écotourisme, plus particulièrement la stratégie québécoise des aires protégées, la stratégie québécoise sur la biodiversité, la stratégie de marketing touristique 2000-2005 du Québec ; les plans régionaux de développement du territoire public (PRDTP) du MRN ; les processus des plans de développement régional associés aux ressources fauniques (PDRRF) ${ }^{5}$ qui sont mis de l'avant par la Société de la faune des parcs du Québec dans toutes les régions de la province ; les plans des conseils régionaux de l'environnement (CRÉ), les ententes de développement entre les régions et le gouvernement et les stratégies de marketing des ATR.

Le secteur de l'écotourisme, dominé par les petites entreprises artisanales, se caractérise par un taux important de roulement de l'offre et rend souvent difficile l'accès au financement. L'organisation des producteurs à l'échelle régionale demeure peu ou pas structurée.

Les efforts actuels de promotion des produits d'écotourisme en sont à leurs balbutiements. Toutefois, des outils promotionnels totalement ou partiellement dédiés à l'écotourisme sont récemment apparus. Globalement, l'utilisation explicite du terme écotourisme demeure encore peu répandue dans les entreprises, sur une base individuelle, mais une proportion importante d'entreprises souhaiterait développer une offre écotouristique ou considèrent offrir des produits d'écotourisme. Finalement, le Québec comme destination est peu présent et les producteurs qui ont leur siège social au Québec sont totalement absents des sites Internet de référence majeurs (i-Explore, Specialty Travel Index, Gorp, Green-Travel). Les efforts de marketing se révèlent complexes, compte tenu du caractère hétérogène du marché.

\section{Enjeux et défis :}

- Bonne organisation du secteur sur laquelle le Québec peut capitaliser;

- Positionnement des produits d'écotourisme québécois sur les sites Internet de référence majeurs;

- Consolidation du réseau des producteurs professionnels et d'Aventure Écotourisme Québec;

- Amélioration de « l'image d'affaires » du secteur;

- Identification des besoins de formation et de développement d'une offre de formation adaptée;
- Accès à une main-d'œuvre compétente, à de la formation et du perfectionnement en région;

- Amélioration de l'accès, notamment aérien, aux régions éloignées et aux sites isolés;

- Soutien au développement des produits (ex. : coût souvent trop élevé en repérage sur le terrain lors de la création de produits);

- Développement d'une meilleure connaissance des marchés;

- Support et mise en marché distinctive des produits d'écotourisme certifiés par le «Programme Qualité ».

Ce premier diagnostic de l'écotourisme au Québec, qui est disponible sur le site Internet de Tourisme Québec (www.tou risme.gouv.qc.ca), servira de base de réflexion à un symposium ainsi qu'à la production d'une stratégie au cours de 2003.

\section{Maurice Couture est consultant spé-} cialisé en tourisme (nature, aventure, écotourisme, autochtone) et il est responsable du cours "Écotourisme et espaces naturels $» \grave{a} l ' U Q A M$.

\section{Notes}

1 Ministère de l'Environnement du Québec, Consultation sur le projet de Stratégie québécoise sur la diversité biologique 20022007: Québec se mobilise en vue d'une nouvelle stratégie sur la biodiversité http://www.menv.gouv.qc.ca/biodiversite/ strateg_02-07/consultation.htm.

2 On pourra consulter le texte intégral sur le site http://www.tourisme.gouv.qc.ca/fran cais/idees_vac/bonne_conduite.html.

3 http://www.baleinesendirect.com.

4 Article du $D^{r}$ Alice Crabtree, TIES Newsletter, $3^{\circ}$ trimestre, 2000.

5 http://www.fapaq.gouv.qc.ca /fr/PDRRF/index.htm.

\section{Bibliographie}

Couture, Maurice (2002), Nature et tourisme : L'Écotourisme au Québec en 2002, Tourisme Québec. 